\title{
Investigation of very low Earth orbits (VLEOs) for global spaceborne lidar
}

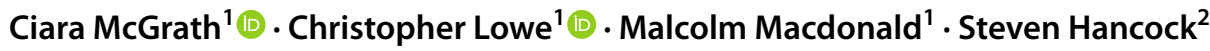

Received: 27 August 2021 / Revised: 23 December 2021 / Accepted: 28 January 2022 / Published online: 22 February 2022

(c) The Author(s) 2022

\begin{abstract}
Very low Earth orbits (VLEOs) have been proposed as a beneficial space mission regime due to their propensity to increase instrument spatial resolution and reduce launch cost per unit mass. However, for visual instruments, these benefits come at the cost of a decreased instrument swath width. This reduction results in longer revisit periods for regions on Earth and longer time until global coverage is achieved. Conversely, light detection and ranging (lidar) as an active remote sensing technique, can benefit from larger swath widths at lower altitudes, due to the increased signal-to-noise ratio. Investigation of this relationship shows that lidar swath width is inversely proportional to altitude squared, and, as a result, the number of spacecraft required to provide a desired lidar coverage also decreases approximately in inverse proportion to altitude squared. Investigation of suitable propulsion systems shows that although propellant mass and number of thrusters required for orbit maintenance increases with decreasing altitude, the overall system mass, and hence launch cost, will, in general, tend to decrease with decreasing altitude due to the lower number of spacecraft required. For a given mission, spacecraft bus, and propulsion system, a VLEO altitude can be identified that will result in the minimum overall mission cost.
\end{abstract}

Keywords Very low Earth orbit $\cdot$ Lidar $\cdot$ Satellite constellation $\cdot$ Space mission analysis

\section{Introduction}

Light detection and ranging (lidar) is a remote sensing method used to examine the surface of the Earth. Using a pulsed laser, lidar uses ranging measurements of the Earth to generate very precise topographical information, such as bare Earth elevation and the structure of vegetation $[1,2]$. Airborne lidar has been used by governments and businesses

Ciara McGrath

ciara.mcgrath@manchester.ac.uk

Christopher Lowe

christopher.lowe@strath.ac.uk

Malcolm Macdonald

malcolm.macdonald.102@strath.ac.uk

Steven Hancock

steven.hancock@ed.ac.uk

1 Applied Space Technology Laboratory (ApSTL), Department of Electronic and Electrical Engineering, University of Strathclyde, 204 George St, Glasgow G1 1XW, Scotland, United Kingdom

2 School of Geosciences, University of Edinburgh, Crew Building, Edinburgh EH9 3FF, Scotland, United Kingdom to make maps for use in flood modelling, carbon content mapping, and investigation of archaeological sites, among others [3-5]. However, airborne lidar requires the dedicated flight of a lidar equipped aircraft over the region of interest. As such, it has a high cost per unit area and is impractical for global mapping.

Spaceborne lidar has the potential to provide global lidar mapping through continuous observation from orbit. Previous spaceborne lidar missions such as ICESat [6], ICESat-2 [7], CALIPSO [8], CATS [9], Aeolus [10], and GEDI [2] have demonstrated the ability of lidar to generate high precision measurements of a variety of phenomena, from ice-cap volume, to clouds, and forests. Irrespective of application, all lidar missions to date have struggled to obtain continuous global measurements. The high power requirement of spaceborne lidar, even compared to other active remote sensing techniques such as radar, results in spaceborne lidars sampling very small areas. GEDI [2] has the widest swath of existing spaceborne lidar and is hosted on the International Space Station (ISS) providing a reliable high power source. Even so, during its two year mission, GEDI's sparse sampling will result in only around 2-4\% of the Earth's land surface being directly imaged. 
Very low Earth orbits (VLEOs) are generally understood to be low-altitude orbits in which the residual atmosphere affects spacecraft significantly, particularly in terms of drag and atomic oxygen erosion. VLEOs have been proposed as a beneficial regime to enable fast natural spacecraft deorbit and reduce space debris, as well as to improve spatial resolution of instruments. The increased atmospheric density at VLEO altitudes poses challenges such as increased atmospheric drag and disturbance forces, however, work is ongoing to address these challenges through the development of air-breathing propulsion systems [11, 12], aerodynamic control surfaces [13], and aerodynamic materials [14]. No consistent definition of VLEO orbits has been agreed upon, with upper bounds of $300 \mathrm{~km}, 450 \mathrm{~km}$, and $500 \mathrm{~km}$ commonly suggested [15-17]. For the purposes of this work an upper limit of $400 \mathrm{~km}$ is used.

VLEO is a potentially advantageous regime for spaceborne lidar desiring high, or even global, coverage. At lower altitudes, the energy that must be emitted by the lidar to achieve sufficient detected energy at the receiver, is reduced [18]. A lidar instrument designed for operation at lower altitudes can hence achieve the same spatial resolution as those at higher altitudes, while allowing the laser power to be spread over a wider swath. Because of this, VLEOs offer the possibility to significantly decrease the number of spacecraft required to achieve desired lidar coverage in a given time. This has been previously noted in [19], but the potential reductions in spacecraft numbers and mission cost have not been investigated in detail. Ref. [17] has shown a potential reduction in launch mass for high resolution optical and synthetic aperture radar (SAR) spacecraft systems operated in VLEO. In this work, analytical descriptions of spaceborne lidar performance are used to provide insights into overall trends and investigate whether a similar relationship exists for lidar. The benefits of increased swath width are weighed against the challenges of maintaining the VLEO altitude for a sufficient mission duration and, to quantify the insights obtained, an example lidar mission requiring near global coverage is used to estimate potential trends in mission launch costs as a function of varying altitude.

\section{Spaceborne lidar performance as a function of altitude}

\subsection{Swath width}

The swath width of a spaceborne lidar can be calculated by considering the lidar performance and the spacecraft orbital velocity. The lidar equation can be combined with the orbital velocity equation to express the swath width as a function of orbit altitude as [18]
$s=\frac{P_{\text {pay }} L_{e}}{E_{\text {det }}} \frac{A}{2 \pi h^{2}} Q \rho \tau^{2} \frac{r^{2}(R+h)^{\frac{3}{2}}}{R \sqrt{\mu}}$,

where $s$ is the swath width, $P_{p a y}$ is the power available to the lidar payload from the spacecraft bus, $L_{e}$ is the laser efficiency, $E_{\text {det }}$ is the energy detected at the receiver (after detector efficiency losses), $A$ is the telescope collecting area, $h$ is the orbit altitude, $\tau$ is the atmospheric transmittance, $Q$ is the detector quantum efficiency, $\rho$ is the surface reflectance, $R$ is the mean Earth radius, $r$ is the desired spatial resolution of the instrument, and $\mu$ is the standard gravitational parameter of Earth. A full derivation of equation (1) is provided in [18]. This approach uses the lidar equation to determine the amount of energy needed per pulse for an accurate measurement. This is then multiplied by the frequency that pixels pass under the satellite as it orbits to give the power needed per ground track to achieve continuous lidar coverage, from the satellite ground speed (which is a function of altitude). This power per track can then be divided by the power available to the laser multiplied by the laser efficiency to define the number of across-track pulses that can be produced, and so the resulting swath width.

Assuming all payload and platform dependent parameters are constant, equation (1) can be arranged to show that

$s \propto \frac{(R+h)^{3 / 2}}{R h^{2}}$.

If $h \ll R$, the numerator can be approximated as $(R+h)^{3 / 2} \approx R^{3 / 2}$ such that

$s \propto \frac{1}{h^{2}}$

with an error $<10 \%$ for $h<500 \mathrm{~km}$ and $R=6371 \mathrm{~km}$. Equation (3) demonstrates that if designing a spaceborne lidar with a fixed resolution and power, the largest swath width can be achieved at the lowest orbital altitude, due to the approximately inverse square relationship.

Using the constants and sample parameters in Table 1, the expected variation in lidar swath width as a function of altitude can be calculated using equation (1) as shown in Fig. 1. The parameters given in Table 1 are based on those of GEDI [2] to give a representative example of current spaceborne lidar capabilities. However, the optics area and payload power are adjusted to values that could be suitable for a free-flying spacecraft in VLEO, with further justification of these parameters in Section 3. These results show the expected swath width for a range of altitudes from $200 \mathrm{~km}-400 \mathrm{~km}$ and for a desired spatial resolution of $5 \mathrm{~m}$, $10 \mathrm{~m}, 20 \mathrm{~m}$, and $30 \mathrm{~m}$. 
Table 1 Sensitivity analysis parameters

\begin{tabular}{llll}
\hline Parameter & Symbol & Value & Unit \\
\hline Mean Earth radius & $R$ & 6371 & $\mathrm{~km}$ \\
Gravitational parameter of Earth & $\mu$ & $3.986 \times 10^{14}$ & $\mathrm{~m}^{3} / \mathrm{s}^{2}$ \\
Surface reflectance & $\rho$ & 0.4 & - \\
Atmospheric transmittance & $\tau$ & 80 & $\%$ \\
Energy detected at receiver & $E_{\text {det }}$ & 0.562 & $\mathrm{fJ}$ \\
\% quantum efficiency & $Q$ & 45 & $\%$ \\
Payload Power & $P_{\text {pay }}$ & 150 & $\mathrm{~W}$ \\
Laser efficiency & $L_{e}$ & 8 & $\%$ \\
Optics area & $A$ & 0.24 & $\mathrm{~m}^{2}$ \\
\hline
\end{tabular}

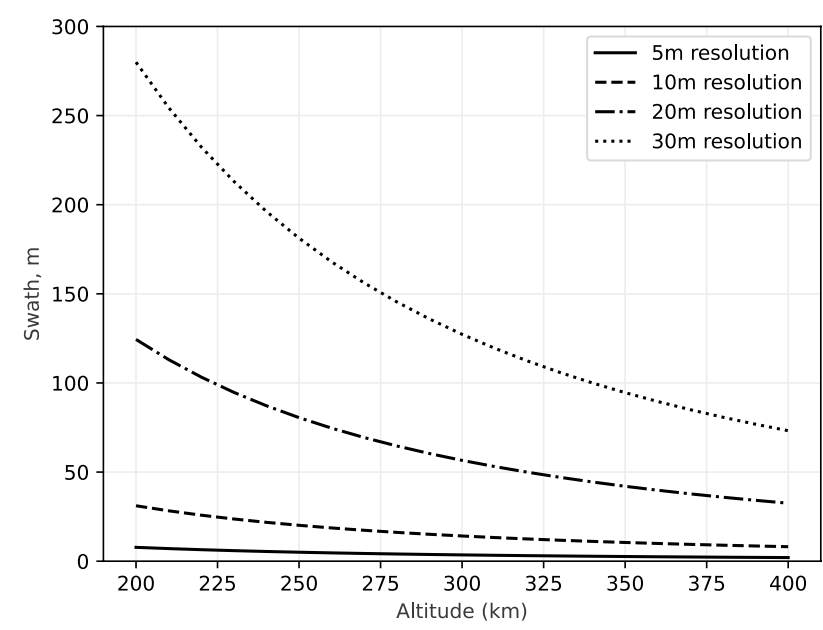

Fig. 1 Lidar swath width as a function of orbit altitude and lidar resolution

\subsection{Resolution}

Given the relationship between swath width and resolution in equation (1), it would be mathematically possible to produce a solution in which the swath width was narrower than the spatial resolution. In reality, however, this would not be possible as there needs to be a minimum energy per laser shot for an accurate measurement $\left(E_{\text {det }}\right)$ and so the satellite would not be able to provide sufficient power to maintain continuous along-track coverage. To avoid this, a minimum resolution limit $\left(r_{\min }\right)$ is defined that will ensure that a continuous track at least one pixel wide with $E_{\text {det }}$ per shot can be produced. This is detailed in [18] and can be calculated by using equation (1) and letting $s=r=r_{\min }$, giving

$r_{\text {min }}=\frac{2 \pi E_{d e t} \sqrt{\mu}}{Q A \rho \tau^{2}} \frac{1}{P_{p a y} L_{e}} \frac{R h^{2}}{(R+h)^{3 / 2}}$.

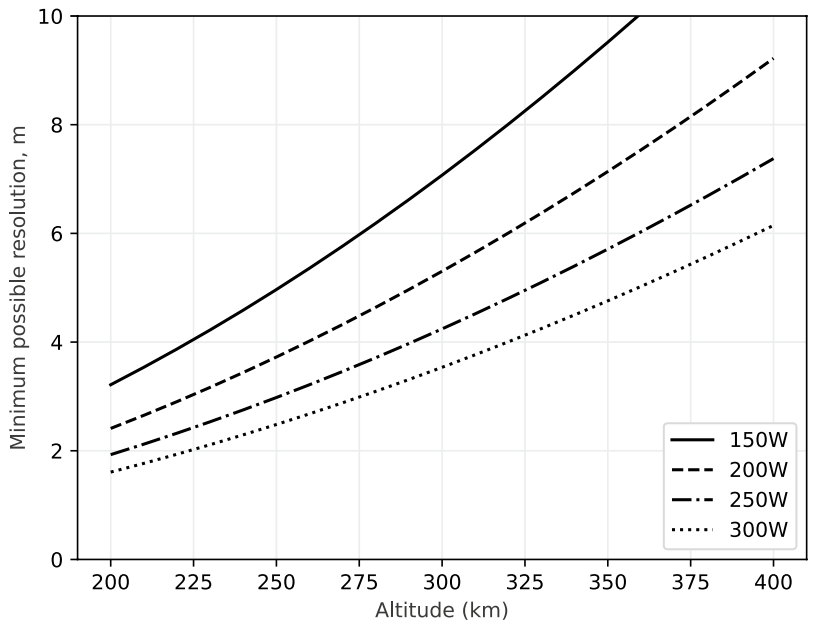

Fig. 2 Minimum possible resolution for the lidar instrument as a function of orbit altitude and payload power. The corresponding swath width for these minimum resolutions would be exactly one resolution element wide such that $s=r_{\min }$

Assuming that the lidar parameters remain constant, the minimum resolution can be seen to vary as a function of altitude as

$r_{\text {min }} \propto \frac{h^{2}}{(R+h)^{3 / 2}}$.

Assuming, as before, that $h \ll R$, this can be approximated to

$r_{\min } \propto h^{2}$,

with an error $<10 \%$ for $h<500 \mathrm{~km}$. Equation (6) demonstrates that lower resolutions (improved definition) can be achieved at lower altitudes. Using the parameters in Table 1, this variation is shown in Fig. 2 for a range of payload powers. This shows that the improvement in minimum resolution as a function of altitude is proportionally more significant for lower payload powers. Furthermore, for a given set of lidar parameters and payload powers, there will be a limit in the resolution that can be achieved unless VLEO altitudes are used. For the example in Fig. 2, it is estimated that a system with a payload power of approximately $150 \mathrm{~W}$ would need to be operated at an altitude of $250 \mathrm{~km}$ or lower to obtain a resolution of $5 \mathrm{~m}$.

\subsection{Number of spacecraft}

To estimate the number of spacecraft needed to meet a defined coverage requirement (assuming that the spacecraft cannot manoeuvre and are in non-repeating ground-tracks) the circumference of the largest latitude band to be covered 
$\left(c_{\max }\right)$ should be identified. For global coverage, $c_{\max }$ will be the circumference of the equator.

The number of orbit revolutions required to provide full coverage of the largest latitude band, assuming no overlap between passes, can be estimated as

$N_{\text {rev }}=\frac{c_{\max }}{2 W}$,

where $W$ is the width of the latitude band covered by a single pass of the spacecraft and noting that a single revolution will provide two passes of the latitude: an ascending and a descending pass. The width of the latitude band covered by a spacecraft in a single pass is calculated as

$W=\frac{s}{\sin \beta}$,

where

$\beta=\mid \tan ^{-1}\left(\frac{\sqrt{\sin ^{2} i-\sin ^{2} \delta}}{\cos i-\omega \cos ^{2} \delta}\right)$,

and $\delta$ is the latitude of interest, $\omega$ is the rotational rate of the Earth, taken as $7.29212 \times 10^{-5} \mathrm{rad} / \mathrm{s}$ and $i$ is the orbit inclination [20]. It should be noted that as the orbit inclination approaches $0 \mathrm{deg}, W$ will tend to infinity. As such, the above approximation is not valid at inclinations close to 0 . Furthermore, due to the narrow swath width of the lidar instrument (section 2.1), latitude bands for which $|\delta|>\left|\tan ^{-1}(\tan i)\right|$ will not be viewed and orbit inclinations should be selected accordingly to ensure the desired coverage ${ }^{1}$.

Assuming that full coverage of the largest latitude band will ensure full coverage of all visible latitude bands, the minimum number of spacecraft required to provide the desired coverage in a given time can be calculated as

$N_{\text {sats }} \geq\left\lceil\frac{N_{\text {rev }}}{\left(1-f_{\text {cloud }}\right)} \frac{T}{t},\right\rceil$

where $f_{\text {cloud }}$ is the assumed global mean cloud fraction, such that $0 \leq f_{\text {cloud }} \leq 1$. It should be noted that this is an approximation of the impact of cloud cover, and more precise calculations of the probability, as in [18], could be used without impacting the presented approach. The total time to desired coverage in seconds is $t$, and $T$ is the satellite orbit period, calculated for circular orbits as

$T=2 \pi \sqrt{\frac{(h+R)^{3}}{\mu}}$.

$\overline{1}$ The $\tan ^{-1}(\tan i)$ function is used here to account for prograde orbits, in which $i>\frac{\pi}{2}$.

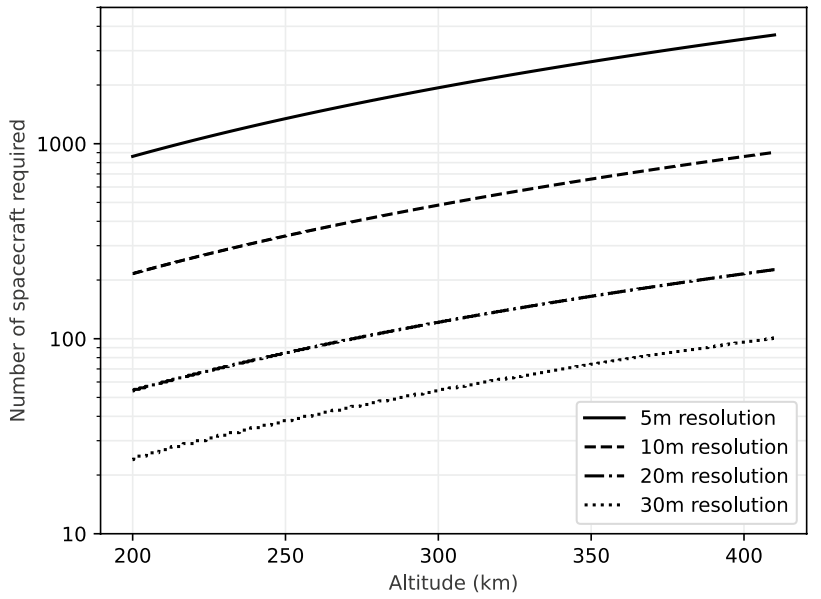

Fig. 3 Number of spacecraft in sun-synchronous orbits required for lidar coverage in one year as a function of orbit altitude for a variety of resolutions (logarithmic scale). The stepped lines are a result of requiring solutions with whole numbers of spacecraft

Combining equations (1) and (7) - (11) allows the minimum number of spacecraft required to achieve the desired coverage in a given time to be calculated as

$N_{\text {sats }} \geq\left\lceil h^{2} \frac{\sin \beta}{t r^{2}} \frac{E_{\text {det }}}{P_{\text {pay }} L_{e} A Q \rho \tau^{2}} \frac{2 \pi^{2} c_{\text {max }} R}{\left(1-f_{\text {cloud }}\right)}\right\rceil$,

noting that this is the minimum possible number that could be required, assuming no overlap between spacecraft swaths. Equation (12) shows that the number of spacecraft required is proportional to the orbit altitude squared, assuming all other variables remain constant. For spacecraft in sun-synchronous orbits, the inclination can be calculated as

$i_{s s}=\cos ^{-1}\left[\frac{-2(h+R)^{7 / 2} \dot{\Omega}_{s s}}{3 R^{2} J_{2} \sqrt{\mu}}\right]$,

where $\dot{\Omega}_{s s}$ is the rate of nodal drift needed for a sun-synchronous orbit, taken as $1.991063853 \times 10^{-7} \mathrm{rad} / \mathrm{sec}$, and $J_{2}$ is the coefficient of the Earth's gravitational zonal harmonic of the 2nd degree, taken as $1082.7 \times 10^{-6}$. Knowing that the inclination of a sun-synchronous orbit will vary only slightly as a function of altitude $\left(96.3 \mathrm{deg} \leq i_{s s} \leq 97.1 \mathrm{deg}\right.$ for $200 \mathrm{~km}$ $\leq h \leq 400 \mathrm{~km}$ ), it can be assumed that

$N_{\text {sats }} \propto h^{2}$

for spacecraft with fixed inclinations or in sun-synchronous orbits. Using the parameters in Table 1 the minimum number of spacecraft in sun-synchronous orbits required to provide coverage of all visible latitudes in one year as a function of altitude are calculated and shown in Fig. 3 for a global cloud cover fraction of $50 \%$, and a variety of resolutions. 


\subsection{Propulsion}

A vital aspect of any VLEO mission that must maintain its orbit for a prolonged period of time, is the need for propulsion to overcome the effects of atmospheric drag. The mass impact of the propulsion system, and any secondary mass impacts such as those from electrical power system mass increase if electric propulsion is used, must be considered. The following sections discuss propulsion options and their respective mass and power implications on the platform, for a range of VLEO altitudes.

\subsubsection{Propulsion background}

For the purposes of this study, the force from atmospheric drag $\left(F_{D}\right)$ experienced on a spacecraft can be be represented by

$F_{D}=\frac{1}{2} d V^{2} \alpha C_{D}$

where $d$ is the atmospheric density, $V$ is the orbital velocity, $\alpha$ is the cross sectional area of the spacecraft in the velocity direction and $C_{D}$ is the drag coefficient. Orbital velocity can be calculated as

$V=\sqrt{\frac{\mu}{R+h}}$

and, ignoring regional, seasonal and solar-cycle induced variations in atmospheric conditions, atmospheric density $\left(d\right.$, in $\left.\mathrm{kg} / \mathrm{m}^{3}\right)$ can be approximated using a power-law curve fit to the 1976 Standard Atmosphere model [21], such that

$d=\Lambda\left(\frac{h}{1000}\right)^{-\gamma}$,

where $\Lambda=10^{7}, \gamma=7.201$ and $h$ is the orbit altitude (in $\mathrm{km}$ ). Note that this relationship is derived from a power-law curve fit to empirical data, such that the dimensions do not need to match. The propellant mass $\left(M_{p}\right)$ required to maintain a specific orbit for a lifetime duration of $L$ can be calculated as

$M_{p}=\frac{L F_{D}}{g_{0} I_{s p}}$,

where $I_{s p}$ is the specific impulse of the propulsion system being used and $g_{0}$ is the acceleration due to gravity (at sea level), taken as $9.81 \mathrm{~m} / \mathrm{s}^{2}$. Combining equations (15) - (18), the mass of propellant required to maintain the orbit altitude can be written as a function of altitude, as

$M_{p}=\frac{L C_{D} \mu \alpha \Lambda}{2 g_{0} I_{s p}} \frac{1000^{\gamma}}{h^{\gamma}(R+h)}$.

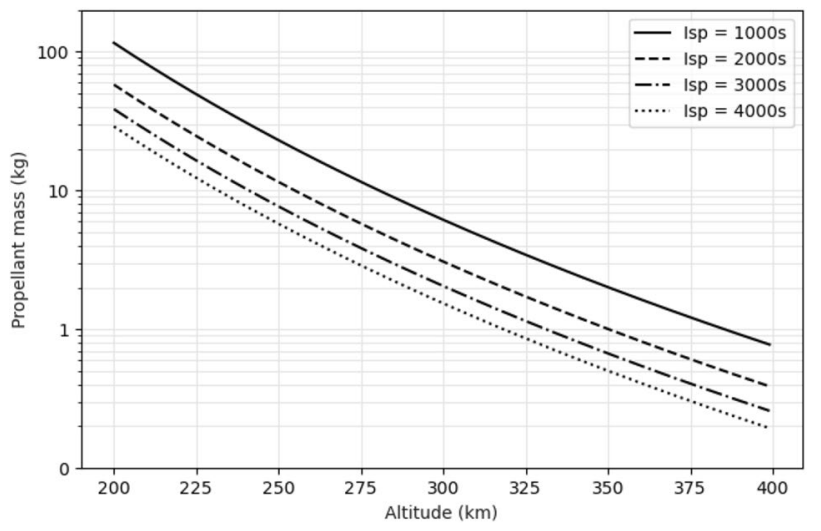

Fig. 4 Propellant mass for different specific impulse performance at altitudes of between $200 \mathrm{~km}$ and $400 \mathrm{~km}$ (logarithmic scale)

If it is once again assumed that $h \ll R$ (i.e. VLEO) it follows that

$M_{p} \propto \frac{1}{h^{\gamma}}$,

such that the mass of propellant required will increase at an increasing rate with decreasing orbit altitude, making orbit maintenance a challenge at very low altitudes. Furthermore, based on the above relationships, attention should be paid to ensuring a high $I_{s p}$ and a low $C_{D}$ in order to minimise propellant mass required. This will have the benefit of either allowing more space for payload or other support systems, or reducing overall mass and therefore launch cost.

\subsubsection{Propulsion system mass}

This, and the following section (2.4.3), illustrate the mass resulting from inclusion of a variety of propulsion systems, and the additional electrical power supply (EPS) mass required to support them, as a function of the mission altitude.

A cross-sectional area of $0.4 \mathrm{~m}^{2}$ and drag coefficient of 2.2 are considered reasonable for this feasibility analysis. The value used for cross-sectional area is derived from a spacecraft of sides between $0.5 \mathrm{~m}$ and $0.8 \mathrm{~m}$ in length, and the drag coefficient, while lower than that demonstrated on other VLEO spacecraft such as GRACE [22], is justifiable for compact, cuboid-like platforms [23]. It is noted that an increase in drag coefficient or cross sectional area would have a proportional impact on the fuel mass required over the mission lifetime, which should be accounted for in any future work.

Using equation 19 , the mass of propellant required to maintain the spacecraft altitude is shown in Fig. 4 for altitudes between $200 \mathrm{~km}$ and $400 \mathrm{~km}$ and specific impulse between 1,000 s and 4,000 s, assuming a 5 year lifetime. Only 
electric propulsion is being considered for this analysis, due to its suitability for continuous, low-thrust precision orbit maintenance. Figure 4 demonstrates that increasing $I_{s p}$ decreases the mass of propellant required at a given altitude.

While the relationships in section 2.4.1 capture the propellant mass required to ensure orbit maintenance for a particular mission lifetime, the capabilities of specific thruster sub-systems must also be considered. The number of thruster units $\left(N_{t h}\right)$ required to overcome the drag force $\left(F_{D}\right)$ can be defined as

$N_{t h}=\max \left(\left\lceil\frac{F_{D}}{F_{\max }}\right\rceil,\left\lceil\frac{L F_{D}}{g_{0} I_{s p} M_{p p u}}\right\rceil\right)$,

where $F_{\max }$ is the maximum thrust force available from a single thruster unit and $M_{p p u}$ is the propellant mass "per unit", i.e. the mass of propellant allocated to each thruster unit. $M_{p p u}$ is not applicable to propulsion systems using a scalable fuel tank, for which this value can be considered infinite for the purposes of this work. Therefore, the total mass of the thruster system $\left(M_{t h}\right)$ is calculated as

$M_{t h}=N_{t h} m_{t h}+M_{p}+M_{t a n k}$

where $m_{t h}$ is the mass of a single thruster head unit (not including the propellant or storage tank, but including all supporting systems required for each unit, such as pipe work, flow control, power distribution units and support structure) and $M_{\text {tank }}$ is the mass of the propellant tank, which can be ignored for systems that have an integrated tank considered as part of the thruster head unit. For this study, a propellant tank mass equal to $50 \%$ of the propellant mass required is used for systems without an integrated tank [24-26]. This is likely to be optimistic in the case of low propellant mass and conservative at higher propellant mass, however in the case of the former, the impact of the propellant tank mass inaccuracy will be negligible.

Assuming that either thrust can be scaled in order to meet the level required to overcome drag, or the thruster can be operated at a duty cycle as required to meet the drag compensation requirement over the long-term, total propulsion system power $\left(P_{T t h}\right)$ demand is calculated as

$P_{T t h}=\frac{F_{D} P_{t h}}{F_{\max }}$

where $P_{t h}$ is the power demand from each thruster unit at maximum thrust, $F_{\max }$.

Given the nature of the consistent low thrust operation required for drag compensation, electric propulsion systems are the most appropriate systems available for VLEO applications. A number of available/experimental propulsion systems have been investigated in terms of suitability, with their respective performance attributes illustrated in Table 2 . The
Table 2 Potential propulsion systems for use in the proposed VLEO lidar mission

\begin{tabular}{|c|c|c|c|c|}
\hline Attribute & T5-GIT & MiXI-ARCH & ENP-R3 & BIT-3 \\
\hline Specific Impulse (s) & 3,500 & 3,200 & 4,000 & 2,150 \\
\hline Thrust (mN) & 20 & 3 & 0.9 & 1.1 \\
\hline Thruster mass (kg) & 27.2 & 0.41 & 2.6 & 1.4 \\
\hline Propellant mass (kg) & N/A & N/A & 1.3 & 1.5 \\
\hline Integrated fuel tank & No & No & Yes & Yes \\
\hline Power demand $^{2}(\mathrm{~W})$ & 585 & 86 & 100 & 75 \\
\hline Flight heritage & Yes & No & Partial $^{1}$ & No \\
\hline References & {$[27,28]$} & {$[29-31]$} & {$[32]$} & {$[33]$} \\
\hline
\end{tabular}

${ }^{1}$ Partial indicates that some aspects of the thruster system have been demonstrated in orbit, but not the complete system.

${ }^{2}$ Power demand is that required by the thruster to operate at full thrust $\left(P_{t h}\right)$

following four thruster systems are considered as potential options, each offering promising characteristics:

1. T5-GIT $[27,28]$ - The T5 gridded ion thruster has flight heritage on board the GOCE and Artemis spacecraft, and provides high thrust and specific impulse performance. It is a larger system compared to the others considered here, designed primarily for platforms of mass $>1000 \mathrm{~kg}$, but could offer a promising solution at the demands of extremely low altitudes, where higher levels of drag compensation are required. The high power demand of $585 \mathrm{~W}$ reflects the relatively high thrust capability, which significantly exceeds that required, even at $200 \mathrm{~km}$ altitude.

2. MiXI-ARCH [29-31] - The Miniature Xenon Ion (MiXI) thruster with Axial Ring Cusp Hybrid (ARCH) discharge is an experimental thruster offering high specific impulse and moderate thrust in a compact, low mass package. While this system has no flight heritage, laboratory experiments show great promise in terms of thrust and specific impulse relative to power demand and size.

3. ENP-R3 [32] - The Enpulsion Micro R3 is a scaledup version of Enpulsion's baseline "NANO" thruster, which uses field emission electric propulsion (FEEP) technology. In comparison to the gridded ion thruster technology used by the T5 and MiXI thrusters, FEEP systems exploit solid fuel, negating the requirement for high pressure storage tanks and associated valve assemblies. Owing to the "complete unit" design, propellant is limited to $1.3 \mathrm{~kg}$ per thruster head as standard, which is assumed to be the case for the analysis carried out in this work.

4. BIT-3 [33] - The Busek Ion Thruster (BIT-3) is a compact, all-in-one, unit providing $\mathrm{mN}$-level thrust at mod- 


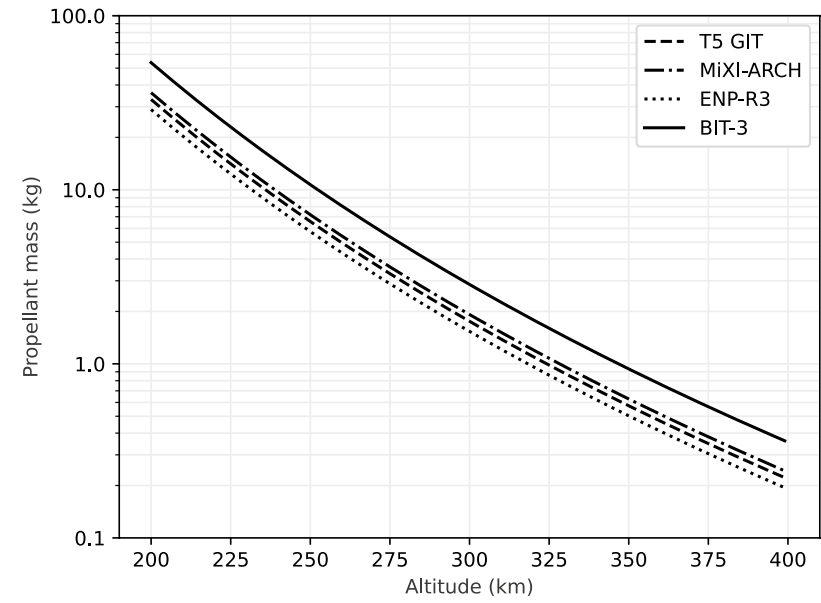

Fig. 5 Propellant mass as a function of orbital altitude (logarithmic scale)

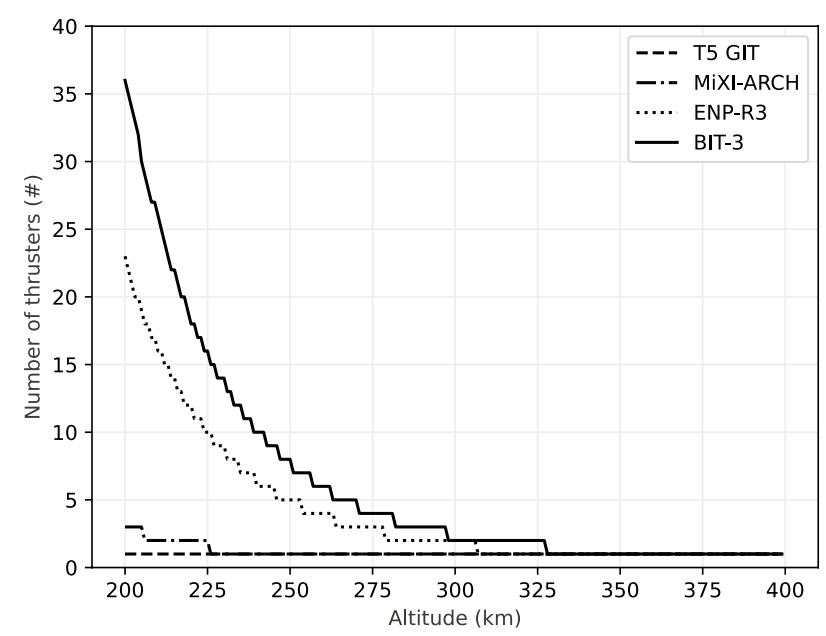

Fig. 6 Required number of thruster units as a function of orbital altitude

erate specific impulse (2150s). It uses iodine as a fuel and, as with the ENP-R3 system, has a self-contained propellant tank, with a $1.5 \mathrm{~kg}$ capacity.

The required propellant mass and number of thruster units for each propulsion system outlined in Table 2 are illustrated in Figs. 5 and 6 respectively, as a function of altitude and assuming a 5 year lifetime. Using equation 22 it is possible to derive the total mass required for each propulsion system, for a range of mission altitudes (Fig. 7).

The power required by each propulsion system as a function of altitude is calculated using equation 23 and shown in Fig. 8. It is clear from this chart that power demand increases significantly at lower altitudes, with all systems investigated here demanding above $100 \mathrm{~W}$ continuous power at orbits

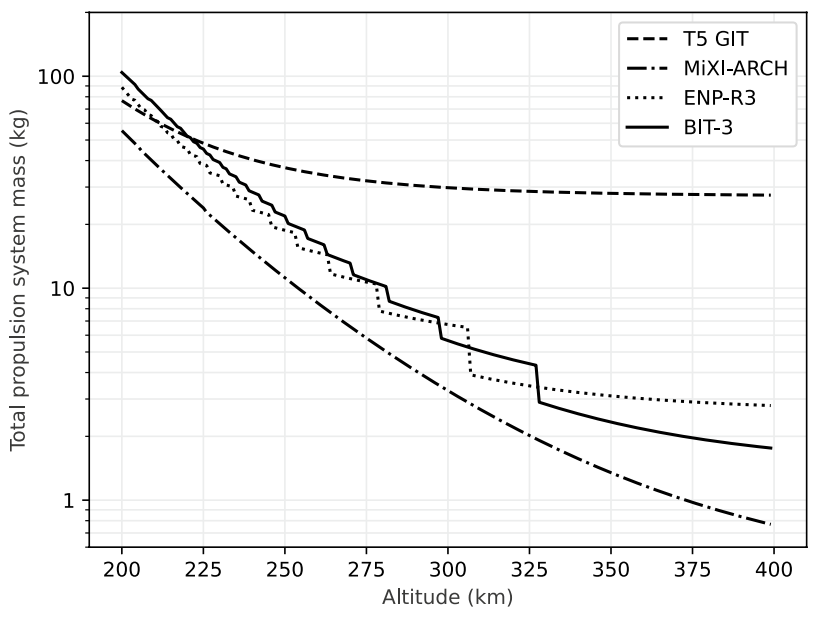

Fig. 7 Total propulsion system mass as a function of orbital altitude (logarithmic scale)

below $220 \mathrm{~km}$. It is noted that a continuous power demand of such magnitude will require additional active cooling, as well as a significant impact on electrical power supply capabilities (sections 2.4.3). This cooling would further impact the overall mass, which would need to be considered carefully in any future analysis.

\subsubsection{Electric power system mass delta}

To meet the power demands from the propulsion system at VLEO, the platform's Electrical Power System (EPS) will need to scale accordingly. This includes an increase in both the battery capacity and solar panel area, accounting for a constant power demand from the propulsion unit throughout both sunlit and eclipse conditions. Given the conceptual nature of this study, the focus is on quantifying the mass impact (delta) from the propulsion system power demand, which represents a change to the nominal power required by the bus. Throughout these calculations, a circular, middaymidnight, Sun-synchronous orbit is considered, such that eclipse conditions are at their worst case (longest), with a duration $38 \%$ of the nominal orbit period [34]. This will represent the most significant EPS mass change.

For the battery, an increase in energy capacity will be required to account for the demand from the propulsion system $\left(P_{T t h}\right)$ during the eclipse period $\left(\tau_{e c l}\right)$. Given a discharge efficiency $\left(\eta_{c h}\right)$, depth of discharge $(D o D)$ and battery specific energy $(\dot{\varepsilon})$ the corresponding change in battery mass can be calculated as:

$\Delta M_{B}=\frac{P_{T t h} \tau_{e c l}}{\dot{\varepsilon} \eta_{c h} D o D}$. 


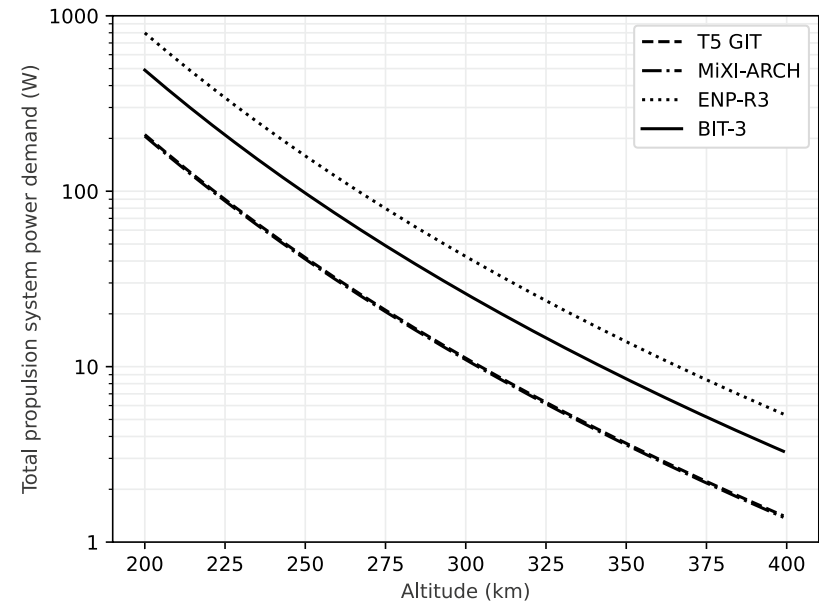

Fig. 8 Total propulsion system power vs. altitude, for different thruster types (logarithmic scale)

The solar panels must compensate for both additional power demand from the propulsion system during a sunlit period $\left(\tau_{\text {sun }}\right)$, and recharging of the battery to offset the energy used by the propulsion system during eclipse. Given solar panel specific power $(\dot{p})$ and efficiencies associated with transfer of power from the solar panels to the sub-systems directly $\left(\eta_{s}\right)$ and via the battery $\left(\eta_{e}\right)$, the increase in solar array mass $\left(\Delta M_{S A}\right)$ can be derived using:

$\Delta M_{S A}=\frac{P_{T t h}}{\dot{p}}\left(\frac{1}{\eta_{s}}+\frac{\tau_{e c l}}{\tau_{\text {sun }} \eta_{e}}\right)$.

The total increase in EPS mass can be approximated by the sum of the increase in battery and solar array mass, assuming a power conditioning and distribution unit (PCDU) that can accommodate the full spectrum of demand across the altitude space. A PCDU such as the AAC Clyde Space Starbuck-Mini [35], which can handle up to $1500 \mathrm{~W}$ of demand, would be suitable. EPS mass delta as a function of altitude is shown in Fig. 9 for a system with the properties given in Table 3 and considering the different propulsion systems identified in section 2.4.2.

\section{Lidar mission size and cost estimation}

As described in section 2.3, the number of spacecraft required to provide a defined lidar coverage can be estimated as a function of altitude using equation (12), with fewer spacecraft needed at lower altitudes. However, the mass of the required thruster system for each spacecraft $\left(M_{t h}\right)$, calculated using equation (22), shows that that $M_{t h}$ will increase with decreasing altitude. Additionally, as described in section 2.4.3 the mass of the power subsystem will also increase with decreasing altitude. As such, to identify the altitude

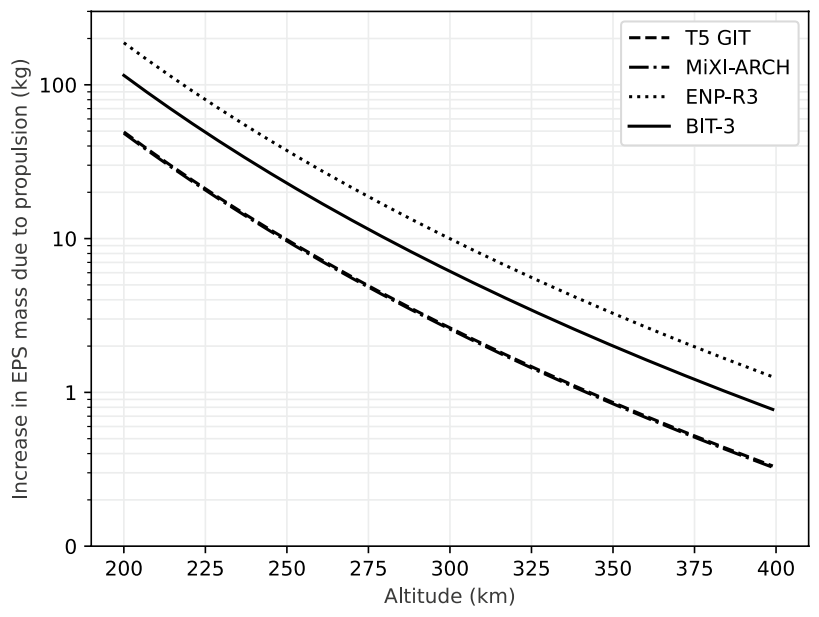

Fig. 9 Increase in Electrical Power System mass vs. altitude, for different thruster types (logarithmic scale)

Table 3 Values used for electrical power system analysis

\begin{tabular}{lllll}
\hline Variable & Symbol & Value & Units & Ref \\
\hline Battery specific energy & $\dot{\varepsilon}$ & 120 & Whr/kg & {$[36]$} \\
Solar array specific power & $\dot{p}$ & 10 & W/kg & {$[37]$} \\
Battery discharge efficiency & $\eta_{c h}$ & 0.75 & - & {$[38]$} \\
$\begin{array}{l}\text { Power delivery efficiency (direct } \\
\quad \text { solar) }\end{array}$ & $\eta_{s}$ & 0.8 & - & {$[38]$} \\
$\begin{array}{l}\text { Power delivery efficiency (via bat- } \\
\quad \text { tery) }\end{array}$ & $\eta_{e}$ & 0.6 & - & {$[38]$} \\
\begin{tabular}{l} 
Battery depth of discharge \\
\hline
\end{tabular} & $D o D$ & 0.8 & - & {$[38]$} \\
\hline
\end{tabular}

at which the lowest total mission mass (and hence lowest launch cost) will occur, the total launch mass for a given mission can be estimated as

$M_{L}=N_{\text {sats }} \times\left(M_{d r y}+M_{t h}+\Delta M_{E P S}\right)$,

where $M_{d r y}$ is the dry mass of the spacecraft and $\Delta M_{E P S}$ is the increase in the mass of the power subsystem due to the requirements of the propulsion system calculated as $\Delta M_{E P S}=\Delta M_{B}+\Delta M_{S A}$, where $\Delta M_{B}$ and $\Delta M_{S A}$ are calculated using equations (24) and (25) respectively. Hence the total launch mass can be calculated as a function of altitude as 


$$
\begin{aligned}
M_{L}= & \left\lceil h^{2} \varepsilon\right\rceil\left\{M_{d r y}+\left[\frac{\zeta \kappa}{h^{\gamma}(R+h)}\right] m_{t h}\right. \\
& +\frac{1}{h^{\gamma}(R+h)}\left[\frac{x \zeta L}{g_{0} I_{s p}}+\frac{\zeta P_{t h}}{F_{\max } \dot{p}}\right. \\
& \left.\left.\left(\frac{\tau_{e c l}}{\dot{\varepsilon} \eta_{c h} D o D}+\frac{1}{\eta_{s}}+\frac{\tau_{e c l}}{\tau_{s u n} \eta_{e}}\right)\right]\right\}
\end{aligned}
$$

where

$\varepsilon=\frac{\sin \beta}{t r^{2}} \frac{E_{\text {det }}}{P_{\text {pay }} L_{e} A Q \rho \tau^{2}} \frac{2 \pi^{2} c_{\text {max }} R}{\left(1-f_{\text {cloud }}\right)}$

$\zeta=\frac{1000^{\gamma}}{2} C_{D} \mu \alpha \Lambda$

$\kappa=\max \left(\frac{1}{F_{\max }}, \frac{L}{g_{0} I_{s p} M_{p p u}}\right)$,

and $x=1$ for a propulsion system with an integrated fuel tank and $x=1.5$ for a propulsion system with no integrated fuel tank.

Assuming a constant launch cost per unit mass $\left(C_{L}\right)$, the total launch cost of the VLEO lidar mission can be estimated as

$C_{\text {total }}=C_{L} M_{L}$.

To investigate how the trends identified will influence the overall estimated cost of a lidar mission, an example mission with the parameters in Table 4 and considering the possible thruster systems in Table 2 is investigated. For the purposes of this trend analysis, and to gain a rough order of magnitude (ROM) insight into potential mission costs, a baseline spacecraft platform dry mass of $150 \mathrm{~kg}$ is considered ${ }^{2}$. This mass is selected with the expectation that future space lidar missions could benefit from significant mass and volume reductions in both payload and platform size as a result of the ever-reducing size of spacecraft electronics, developments in deployable optics, improved laser efficiencies, improved detector efficiencies and improved data processing algorithms [18]. Furthermore, a global lidar mission of this type would not be feasible with significantly larger spacecraft platforms due to the prohibitive costs, and so an assumption is made that for such a mission to be launched, the noted reduction in platform size compared to existing spaceborne lidar must be realised.

The total mass of each spacecraft to be launched as a function of mission altitude is shown in Fig. 10 where an

2 The baseline platform of $150 \mathrm{~kg}$ includes the payload and supporting sub-systems, but not the propulsion system, associated propulsion elements such as propellant and fuel tank, and additional power system mass required to support the propulsion system (e.g. increased solar array area and battery capacity).
Table 4 Example mission parameters

\begin{tabular}{llll}
\hline Parameter & Symbol & Value & Unit \\
\hline Mean Earth radius & $R$ & 6371 & $\mathrm{~km}$ \\
Gravitational parameter of Earth & $\mu$ & $3.986 \times 10^{14}$ & $\mathrm{~m}^{3} / \mathrm{s}^{2}$ \\
Surface reflectance & $\rho$ & 0.4 & - \\
Atmospheric transmittance & $\tau$ & 80 & $\%$ \\
Energy detected at receiver & $E_{\text {det }}$ & 0.562 & $\mathrm{fJ}$ \\
\% quantum efficiency & $Q$ & 45 & $\%$ \\
Payload Power & $P_{\text {pay }}$ & 150 & $\mathrm{~W}$ \\
Laser efficiency & $L_{e}$ & 8 & $\%$ \\
Optics area & $A$ & 0.24 & $\mathrm{~m}^{2}$ \\
Largest latitude to cover (equator) & $\delta$ & 0 & $\mathrm{deg}$ \\
Circumference of largest latitude & $c_{\text {max }}$ & 40075 & $\mathrm{~km}$ \\
Time to full coverage & $t$ & 1 & year \\
Mission lifetime & $L$ & 5 & years \\
Cross-sectional area & $\alpha$ & 0.4 & $\mathrm{~m}^{2}$ \\
Coefficient of drag & $C_{D}$ & 2.2 & - \\
Dry mass & $M_{\text {dry }}$ & 150 & $\mathrm{~kg}$ \\
Cloud fraction & $f_{\text {cloud }}$ & 50 & $\%$ \\
Launch cost per kg [39, 40] & $C_{L}$ & 10,000 & $\$$ \\
\hline
\end{tabular}

${ }^{a}$ At the time of writing, the cost of launch for $150 \mathrm{~kg}$ or $200 \mathrm{~kg}$ spacecraft into low Earth orbit, with Spaceflight Services, is 1,850 kUSD. This represents a cost per kg of $9 \mathrm{kUSD}$ and $6.75 \mathrm{kUSD}$ respectively. A cost of $10 \mathrm{kUSD}$ is therefore used as a conservative estimate

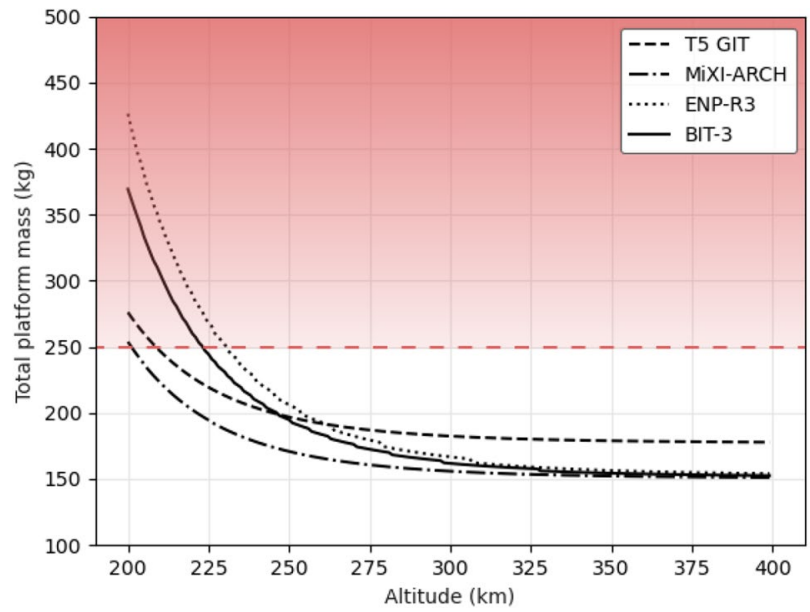

Fig. 10 Total platform mass vs. altitude for different propulsion systems. The region above the red dashed line is considered out of scope in terms of total mass 
upper limit on total platform mass of $250 \mathrm{~kg}$ is imposed to avoid unrealistic configurations. This results in a lower altitude limit of $201 \mathrm{~km}, 209 \mathrm{~km}, 223 \mathrm{~km}$ and $230 \mathrm{~km}$ for the MiXI-ARCH, T5-GIT, BIT-3 and ENP-R3 propulsion systems respectively. Figure 11 shows the estimated launch costs for the mission, assuming a sun-synchronous orbit and a maximum allowable spacecraft mass of $250 \mathrm{~kg}$. These results show that in all cases there is an altitude at which launch cost is minimum, as below this altitude the increased mass of the propulsion and power systems outweighs the benefit of having fewer spacecraft overall. As noted in section 2.4, the high performance of the MiXI-ARCH propulsion system means it outperforms all other propulsion systems in almost all cases. If a propulsion system with flight heritage is desired, the T5-GIT results in lowest launch cost for altitudes below approximately $250 \mathrm{~km}$, while the ENP-R3 system results in a lower launch cost to altitudes greater than approximately $250 \mathrm{~km}$, for the case considered.

The minimum costs and corresponding altitudes and mission parameters for each propulsion system considered are given in Table 5 for a desired spatial resolution of $20 \mathrm{~m}$ and in Table 6 for a desired spatial resolution of $30 \mathrm{~m}$. All values are rounded to the nearest whole number. These results show that the minimum cost solution for the cases examined would be 29 spacecraft capable of providing $30 \mathrm{~m}$ resolution data, each equipped with two MiXI-ARCH thrusters at an altitude of $220 \mathrm{~km}$ requiring $18 \mathrm{~kg}$ of propellant for an overall spacecraft mass of $202 \mathrm{~kg}$ per spacecraft and an estimated cost of $\$ 59 \mathrm{M}$. For a $20 \mathrm{~m}$ spatial resolution, the lowest cost solution would require 64 spacecraft at $218 \mathrm{~km}$ altitude equipped with two MiXI-ARCH thrusters carrying $19 \mathrm{~kg}$ of

Fig. 11 Estimated launch cost for example lidar mission as a function of orbit altitude for a variety of propulsion systems and two spatial resolutions (logarithmic scale). The stepped lines are a result of requiring solutions with whole numbers of thrusters and whole numbers of spacecraft

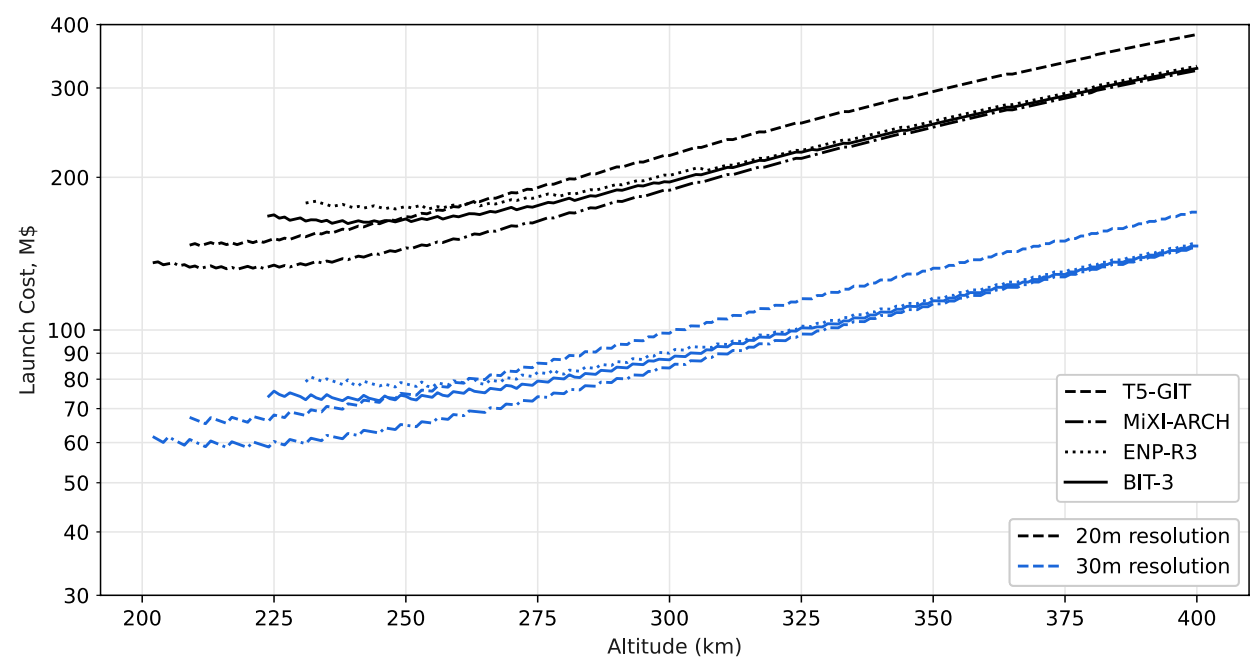

Table 5 Minimum estimated launch costs, corresponding altitudes and mission parameters for selected propulsion system options for a system requiring a $20 \mathrm{~m}$ spatial resolution. $M_{p}$ and $N_{t h}$ refer to the propellant mass and number of thrusters per spacecraft

\begin{tabular}{llllllll}
\hline Prop. system & Altitude & Swath & No. s/c & S/c mass & $M_{p}$ & $N_{\text {th }}$ & Cost \\
\hline T5-GIT & $211 \mathrm{~km}$ & $112 \mathrm{~m}$ & 60 & $244 \mathrm{~kg}$ & $22 \mathrm{~kg}$ & 1 & $\$ 147 \mathrm{M}$ \\
MiXI-ARCH & $218 \mathrm{~km}$ & $105 \mathrm{~m}$ & 64 & $206 \mathrm{~kg}$ & $19 \mathrm{~kg}$ & 2 \\
ENP-R3 & $254 \mathrm{~km}$ & $78 \mathrm{~m}$ & 87 & $199 \mathrm{~kg}$ & $5 \mathrm{~kg}$ & 4 & $\$ 132 \mathrm{M}$ \\
BIT-3 & $239 \mathrm{~km}$ & $88 \mathrm{~m}$ & 77 & $211 \mathrm{~kg}$ & $15 \mathrm{~kg}$ & 10 & $\$ 162 \mathrm{M}$ \\
\hline
\end{tabular}

Table 6 Minimum estimated launch costs, corresponding altitudes and mission parameters for selected propulsion system options for a system requiring a $30 \mathrm{~m}$ spatial resolution. $M_{p}$ and $N_{t h}$ refer to the propellant mass and number of thrusters per spacecraft

\begin{tabular}{lllllll}
\hline Prop. system & Altitude & Swath & No. s/c & S/c mass & $M_{p}$ & $N_{\text {th }}$ \\
\hline T5-GIT & $212 \mathrm{~km}$ & $250 \mathrm{~m}$ & 27 & $242 \mathrm{~kg}$ & $22 \mathrm{~kg}$ & 1 \\
MiXI-ARCH & $220 \mathrm{~km}$ & $232 \mathrm{~m}$ & 29 & $202 \mathrm{~kg}$ & $18 \mathrm{~kg}$ & 2 \\
ENP-R3 & $255 \mathrm{~km}$ & $174 \mathrm{~m}$ & 39 & $198 \mathrm{~kg}$ & $5 \mathrm{~kg}$ & 4 \\
BIT-3 & $245 \mathrm{~km}$ & $188 \mathrm{~m}$ & 36 & $202 \mathrm{~kg}$ & $12 \mathrm{~kg}$ & 9 \\
\hline
\end{tabular}


propellant for an overall spacecraft mass of $206 \mathrm{~kg}$ and an estimated launch cost of $\$ 132 \mathrm{M}$.

\section{Conclusions}

Analysis of analytical expressions describing spaceborne lidar performance can provide insight into the variation in expected performance and mission launch cost as a function of altitude. The swath width of a spaceborne lidar is inversely proportional to the square of the spacecraft altitude, meaning that a lidar spacecraft at a lower altitude will have greater coverage than a similar instrument at a higher altitude. The minimum resolution that can be achieved by a given lidar instrument is approximately proportional to altitude squared, meaning that there is a limit to the spatial resolution that can be achieved at a given altitude. The number of spaceborne lidar required to obtain a desired coverage is directly proportional to altitude squared for a defined set of system parameters. Although the number of spacecraft required for a lidar mission will decrease with decreasing altitude, a turning point may be seen beyond which the system mass, and corresponding mission launch cost, will increase. In these cases, for a spacecraft with a defined set of lidar and platform parameters, an altitude can be identified that will result in a minimum mission cost. To operate in very low Earth orbits (VLEOs) may require additional technologies, such as high quality pointing subsystems, which could increase system mass and cost. However, use of propulsion systems and spacecraft buses optimised for operation in VLEO could improve performance. Furthermore, operation at VLEO altitudes reduces spacecraft numbers and ensures natural orbit decay after the mission is concluded, reducing the potential impact of the system on the orbital debris environment. Initial high level examination has shown that VLEO is a promising option for spaceborne lidar systems requiring significant levels of Earth coverage.

\begin{abstract}
Author contributions All authors contributed to the study conception and design. Analysis was performed by CM and CL. The first draft of the manuscript was written by CM and CL and all authors commented on previous versions of the manuscript. SH was the Principal Investigator on the project and provided the lidar background and equations. $\mathrm{SH}$ and MM reviewed all analysis, results and manuscript drafts. All authors read and approved the final manuscript.
\end{abstract}

Funding This work was funded by the UK Space Agency's National Space Innovation Programme, grant number NSIP20_N08.

Availability of data and materials Not applicable.

Code availability Code used to generate the results in this article is available at https://github.com/ciaramcgrath91/VLEO_journal.

\section{Declarations}

Conflict of interest The authors have no conflicts of interest to declare that are relevant to the content of this article.

Ethics approval Not applicable.

Consent to participate Not applicable.

Consent for publication Not applicable.

Open Access This article is licensed under a Creative Commons Attribution 4.0 International License, which permits use, sharing, adaptation, distribution and reproduction in any medium or format, as long as you give appropriate credit to the original author(s) and the source, provide a link to the Creative Commons licence, and indicate if changes were made. The images or other third party material in this article are included in the article's Creative Commons licence, unless indicated otherwise in a credit line to the material. If material is not included in the article's Creative Commons licence and your intended use is not permitted by statutory regulation or exceeds the permitted use, you will need to obtain permission directly from the copyright holder. To view a copy of this licence, visit http://creativecommons.org/licenses/by/4.0/.

\section{References}

1. Sun, G., Ranson, K., Kimes, D., Blair, J., Kovacs, K.: Forest vertical structure from glas: An evaluation using lvis and srtm data. Remote Sensing of Environment 112(1), 107-117 (2008). https:// doi.org/10.1016/j.rse.2006.09.036

2. Dubayah, R., Blair, J.B., Goetz, S., Fatoyinbo, L., Hansen, M., Healey, S., Hofton, M., Hurtt, G., Kellner, J., Luthcke, S., et al.: The global ecosystem dynamics investigation: High-resolution laser ranging of the earth's forests and topography. Science of Remote Sensing 1, 100002 (2020). https://doi.org/10.1016/j.srs. 2020.100002

3. Coles, D., Yu, D., Wilby, R.L., Green, D., Herring, Z.: Beyond "flood hotspots": modelling emergency service accessibility during flooding in york, uk. Journal of hydrology 546, 419-436 (2017). https://doi.org/10.1016/j.jhydrol.2016.12.013

4. Hudak, A.T., Strand, E.K., Vierling, L.A., Byrne, J.C., Eitel, J.U., Martinuzzi, S., Falkowski, M.J.: Quantifying aboveground forest carbon pools and fluxes from repeat lidar surveys. Remote Sensing of Environment 123, 25-40 (2012). https://doi.org/10.1016/j.rse. 2012.02.023

5. Banaszek, Ł, Cowley, D.C., Middleton, M.: Towards national archaeological mapping. assessing source data and methodology-a case study from scotland. Geosciences 8(8), 272 (2018). https:// doi.org/10.3390/geosciences8080272

6. Schutz, B.E., Zwally, H.J., Shuman, C.A., Hancock, D., DiMarzio, J.P.: Overview of the icesat mission. Geophysical Research Letters 32(21) (2005). https://doi.org/10.1029/2005GL024009

7. Markus, T., Neumann, T., Martino, A., Abdalati, W., Brunt, K., Csatho, B., Farrell, S., Fricker, H., Gardner, A., Harding, D., et al.: The ice, cloud, and land elevation satellite-2 (icesat-2): science requirements, concept, and implementation. Remote sensing of environment 190, 260-273 (2017). https://doi.org/10.1016/j.rse. 2016.12.029

8. Winker, D.M., Vaughan, M.A., Omar, A., Hu, Y., Powell, K.A., Liu, Z., Hunt, W.H., Young, S.A.: Overview of the calipso mission and caliop data processing algorithms. Journal of Atmospheric 
and Oceanic Technology 26(11), 2310-2323 (2009). https://doi. org/10.1175/2009JTECHA1281.1

9. McGill, M.J., Yorks, J.E., Scott, V.S., Kupchock, A.W., Selmer, P.A.: The cloud-aerosol transport system (cats): A technology demonstration on the international space station. In: Lidar Remote Sensing for Environmental Monitoring XV, vol. 9612, p. 96120 (2015). https://doi.org/10.1117/12.2190841. International Society for Optics and Photonics

10. Stoffelen, A., Marseille, G., Bouttier, F., Vasiljevic, D., De Haan, S., Cardinali, C.: Adm-aeolus doppler wind lidar observing system simulation experiment. Quarterly Journal of the Royal Meteorological Society: A journal of the atmospheric sciences, applied meteorology and physical oceanography 132(619), 1927-1947 (2006). https://doi.org/10.1256/qj.05.83

11. Romano, F., Massuti-Ballester, B., Binder, T., Herdrich, G., Fasoulas, S., Schönherr, T.: System analysis and test-bed for an atmosphere-breathing electric propulsion system using an inductive plasma thruster. Acta Astronautica 147, 114-126 (2018). https://doi.org/10.1016/j.actaastro.2018.03.031

12. Wang, Z., Eun, Y., Wu, X.: Design and demonstration of a micro air-fed magnetoplasmadynamic thruster for small satellites. Acta Astronautica 181, 482-491 (2021). https://doi.org/10.1016/j.actaa stro.2021.01.047

13. Roberts, P.C., Crisp, N.H., Edmonson, S., García-Almiñana, D., Rodríguez Donaire, S.: Discoverer-radical redesign of earth observation satellites for sustained operation at significantly low altitudes. In: 68th IAC International Astronautical Congress, Adelaide, Australia, 25-29 Setember 2017, pp. 1-9 (2017)

14. Roberts, P.C., Crisp, N.H., Romano, F., Herdrich, G., Oiko, V., Edmondson, S., Haigh, S., Huyton, C., Livadiotti, S., Lyons, R., et al.: Discoverer-making commercial satellite operations in very low earth orbit a reality. In: Proceedings of the International Astronautical Congress, IAC, vol. 2019 (2019). IAF

15. Walsh, J., Berthoud, L., Allen, C.: Drag reduction through shape optimisation for satellites in very low earth orbit. Acta Astronautica 179, 105-121 (2021). https://doi.org/10.1016/j.actaastro. 2020.09.018

16. Leomanni, M., Garulli, A., Giannitrapani, A., Scortecci, F.: Propulsion options for very low earth orbit microsatellites. Acta Astronautica 133, 444-454 (2017). https://doi.org/10.1016/j.actaa stro.2016.11.001

17. Crisp, N., Roberts, P., Romano, F., Smith, K., Oiko, V., Sulliotti-Linner, V., Hanessian, V., Herdrich, G., García-Almiñana, D., Kataria, D., et al.: System modelling of very low earth orbit satellites for earth observation. Acta Astronautica 187, 475-491 (2021). https://doi.org/10.1016/j.actaastro.2021.07.004

18. Hancock, S., McGrath, C., Lowe, C., Davenport, I., Woodhouse, I.: Requirements for a global lidar system: spaceborne lidar with wall-to-wall coverage. Royal Society Open Science 8(12), 211166 https://doi.org/10.1098/rsos.211166

19. Crisp, N.H., Roberts, P.C., Livadiotti, S., Oiko, V.T.A., Edmondson, S., Haigh, S., Huyton, C., Sinpetru, L., Smith, K., Worrall, S., et al.: The benefits of very low earth orbit for earth observation missions. Progress in Aerospace Sciences 117, 100619 (2020). https://doi.org/10.1016/j.paerosci.2020.100619

20. Washburn, A.R.: Earth coverage by satellites in circular orbit. Department of operations Research Naval Postgraduate School (2004)

21. Macdonald, M., McInnes, C., Lücking, C., Visagie, L., Lappas, V.J., Erb, S.: Needs assessment of gossamer structures in communications platform end-of-life disposal. In: AIAA Guidance, Navigation, and Control (GNC) Conference, p. 4870 (2013). https:// doi.org/10.2514/6.2013-4870

22. Mehta, P.M., Walker, A., Lawrence, E., Linares, R., Higdon, D., Koller, J.: Modeling satellite drag coefficients with response surfaces. Advances in Space Research 54(8), 1590-1607. https:// doi.org/10.1016/j.asr.2014.06.033 (2014)

23. Walsh, J.A., Berthoud, L.: Reducing spacecraft drag in very low earth orbit through shape optimisation. https://doi.org/10.13009/ EUCASS2017-449 (2017)

24. Tam, W., Jackson, A., Nishida, E., Kasai, Y., Tsujihata, A., Kajiwara, K.: Design and manufacture of the ETS VIII xenon tank. https://doi.org/10.2514/6.2000-3677. https://arc.aiaa.org/doi/abs/ 10.2514/6.2000-3677 (2012)

25. Le Floc'h, C., Aufaure, J., Salome, R., Tschofen, J.: New high pressure tank for xenon storage

26. Collard, T.A., Sheehan, J.P., Gallimore, A.D.: Pressurized xenon propellant management system for the cubeSat ambipolar thruster (2015)

27. Randall, Peter N.,R.A.L., Clark, S.D.: Qinetiq t5 based electric propulsion system and architectural options for future applications. In: Proceedings of the 35th International Electric Propulsion Conference, vol. Georgia Institute of Technology, USA. QinetiQ Space UK (2017)

28. Peter N. Randall, S.D.C. Rhodri A. Lewis, Hall, K.W.: T5 performance, industrialisation and future applications. In: Proceedings of the 36th International Electric Propulsion Conference, vol. University of Vienna, Austria. QinetiQ Space UK (2019)

29. Richard E. Wirz, C.M. Daniel Goebel, Mueller, J.: Development of cathode technologies for a miniature ion thruster. In: 39th AIAA/ASME/SAE/ASEE Joint Propulsion Conference \& Exhibit, vol. Huntsville, Alabama. https://doi.org/10.2514/6.2003-4722. University of California, Los Angeles (2003)

30. Richard E. Wirz, M.G. Juergen Mueller, Marrese, C.: Miniature ion thrusters for precision formation flying. In: 40th AIAA/ ASME/SAE/ASEE Joint Propulsion Conference \& Exhibit, vol. Fort Lauderdale, FL. https://doi.org/10.2514/6.2004-4115. University of California, Los Angeles (2004)

31. Dankongkakul, B., Wirz, R.E.: Miniature ion thruster ring-cusp discharge performance and behavior. Journal of Applied Physics 122(24). https://doi.org/10.1063/1.4995638. University of California, Los Angeles (2017)

32. GmbH, E.: Enpulsion Micro R3 datasheet. Accessed 14 Jun 2021. Available: https://www.enpulsion.com/wp-content/uploads/ ENP2018-002.H-MICRO-Thruster-Product-Overview.pdf (2021)

33. Busek, C.I.: Busek BIT-3. Accessed 14 Jun 2021. Available: https://www.busek.com/bit3 (2021)

34. Bhatt, S., Svecz, A., Alaniz, A., Jang, J.-W.J., Nguyen, L.: Thermally-constrained fuel-optimal iss maneuvers. In: 38th Annual AAS GNC Conference (2015)

35. Space, A.C.: AAC clyde space starbuck-mini datasheet. Accessed on: 07 Dec 2021. Available: https://www.aac-clyde.space/wpcontent/uploads/2021/11/AAC_DataSheet_Starbuck-Mini-updat ed.pdf (2021)

36. Space, A.C.: AAC clyde space optimus battery datasheet. Accessed on: 07 Dec 2021. Available: https://www.aac-clyde. space/wp-content/uploads/2021/10/AAC_DataSheet_Optimus. pdf (2021)

37. Macdonald, M., Badescu, V.: Chapter 10, pp. 249-277. Springer, Heidelberg (2016)

38. Fortescue, P.W.: Chapter 10, pp. 346-354. Wiley, Hoboken (2011)

39. Jones, H.: The recent large reduction in space launch cost. 48th International Conference on Environmental Systems (2018)

40. Spaceflight: spaceflight launch services pricing schedule. Accessed 08 Dec 2021. Available: https://spaceflight.com/prici ng/ (2021)

Publisher's Note Springer Nature remains neutral with regard to jurisdictional claims in published maps and institutional affiliations. 